REVIEW ARTICLE

AFRICAN JOURNAL OF CLINICAL AND EXPERIMENTAL MICROBIOLOGY AJCEM/200922/20913

COPYRIGHT 2009

ISBN 1595-689X VOL 10(2)

AFR. J. CLN. EXPER. MICROBIOL 10(2): 99-116

-http://www.ajol.info/journals/ajcem

\title{
RISK FACTORS, THREATS AND PREVENTION OF HIGHLY PATHOGENIC AVIAN INFLUENZA (HPAI) IN AFRICAN COUNTRIES
}

\author{
${ }^{1}$ Musa*, O. I., $^{1}$ Salaudeen A. G., ${ }^{2}$ Akanbi II, A. A. and ${ }^{1 B}$ Bolarinwa, O. A. \\ Departents of ${ }^{1}$ Epidemiology \& Community Health,and Medical Microbiology, College of \\ Medicine, University of Ilorin, Ilorin, Nigeria \\ *Correspondence: E-mail: mtosh2002@yahoo.com
}

\begin{abstract}
Highly pathogenic avian influenza (HPAI) is a viral disease that affects the digestive, nervous and respiratory systems of all domestic and wild birds with high morbidity and mortality. It is highly contagious disease which can be fatal in humans. The avian influenza viruses (AIVs) are classified as types A, B and C with 15 subtypes of the type A. To date, all disease causing HPAI Viruses belong to $\mathrm{H} 5$ or $\mathrm{H} 7$ subtypes; and affect pigs and humans with the pigs serving as a mixing vehicle for re-assortment of the virus. The domestic ducks get infected without showing clinical signs and serve as a source of infection for domestic poultry.

Outbreaks of HPAl in Europe, Asia and Turkey are reported to be associated, with the presence of wet lands and lakes where migratory birds rest. In some African countries like Nigeria, such wet lands exist with free flying wild birds and domestic ducks visiting and resting. The possible source of introduction into a country could be through importation or smuggling of infected poultry products across the borders and through migratory birds that fly through identified pathways. The status of HPAl in many African countries including Nigeria is still under investigation so that appropriate strategies / measures to prevent introduction of the disease into the country can be implemented and / or strengthened through restriction of importation of poultry and poultry products from high risk countries, effective disease surveillance, functional National Veterinary services, quarantine and community based participatory epidemiological system for HPAI surveillance and control.
\end{abstract}

This article reviewed the global epidemiology and risk factors of HPAI infection in Nigeria and other African countries with emphasis on specific preventive measures that can reduce introduction of the virus into the country and the epidemiological surveillance for case detection / identification, screening and management. This review provides useful information and updates for health workers in tropical countries on the trends of AIVs and HPAI, diagnostic criteria using case definitions for both community and health facility levels and management protocols for confirmed cases as recommended by the World health Organization.

\section{Introduction}

Avian influenza, or "bird flu", is a contagious disease of animals caused by viruses that normally infect only birds and, less commonly, pigs. Avian

forms of disease, distinguished by low and high extremes of virulence. The so- influenza viruses (AIVs) are highly species-specific, but have, on rare occasions, crossed the species barrier to infect humans. In domestic poultry, infection with avian influenza viruses causes two main 
called "low pathogenic" form commonly causes only mild symptoms (ruffled feathers, a drop in egg production) and may easily go undetected. The highly pathogenic form is far more dramatic. It spreads very rapidly through poultry flocks, causes disease affecting multiple internal organs, and has a mortality that can approach $100 \%$, often within 48

hours $(1,2)$. The role of migratory birds in the spread of highly pathogenic avian influenza is not fully understood. Wild waterfowl are considered the natural reservoir of all influenza A viruses. They have probably carried influenza viruses, with no apparent harm, for centuries. They are known to carry viruses of the $\mathrm{H} 5$ and $\mathrm{H} 7$ subtypes, but usually in the low pathogenic form. Considerable circumstantial evidence suggests that migratory birds can introduce low pathogenic H5 and H7 viruses to poultry flocks, which then mutate to the highly pathogenic form. In the past, highly pathogenic viruses have been isolated from migratory birds on very rare occasions involving a few birds, usually found dead within the flight range of a poultry outbreak. This finding long suggested that wild waterfowl are not agents for the onward transmission of these viruses $(2,3)$.

Direct or indirect contact between domestic flocks and wild migratory waterfowl has been implicated as a frequent cause of epidemics in poultry populations. It is generally accepted that migratory waterfowl, most notably wild ducks, are the natural reservoir of AI viruses, which can be transmitted to domestic bird populations and to commercial poultry $(2,4)$.

\section{Avian Influenza Viruses}

Avian influenza virus (AIV) is a member of the family Orthomyxoviridae and genus influenza A. The influenza viruses that constitute this family are classified into type A, B, or C based on the differences between their nucleoprotein and matrix protein Antigens. AIVs belong to type A, which are further categorized into subtypes according to the Antigens of the haemagglutinin $(\mathrm{H})$ and neuraminidase (N) projections on their surfaces. There are 16 haemagglutinin subtypes and 9 Neuraminidase subtypes. Only viruses of the H5 and H7 subtypes are known to cause the highly pathogenic form of the disease. However, not all viruses of the $\mathrm{H} 5$ and $\mathrm{H} 7$ subtypes are highly pathogenic and not all will cause severe disease in poultry. On present understanding, $\mathrm{H} 5$ and $\mathrm{H} 7$ viruses are introduced to poultry flocks in their low pathogenic form. When allowed to circulate in poultry populations, the viruses can mutate, usually within a 
few months, into the highly pathogenic form. This is why the presence of an H5 or $\mathrm{H7}$ virus in poultry is always cause for concern, even when the initial signs of infection are mild $(2,3,5,6)$.

AIVs are susceptible to warm environmental condition, short term exposure to ultraviolet radiation and desiccation. Common detergents and disinfectants such as calcium \& sodium hypochloride and Virlcox can readily inactivate it. However the virus can survives for $35-44$ days in faeces at $4{ }^{\circ} \mathrm{C}$ but for 4 days at $25^{\circ} \mathrm{C}$. Within poultry farms the virus can survive for up to 35 days. AIVs can be isolated from Lake Water where they can persist and retain infectivity for more than 30 days at $0{ }^{\circ} \mathrm{C}$ and for only 4 days at $22 \circ \mathrm{C}(7,8)$. AIVs are shed in faeces and respiratory tract of infected birds for at least 14 days and can be recovered from refrigerated carcasses for 23 days. Birds processed during the viraemic stage can contaminate other carcasses with blood and faeces containing the virus. Eggs laid in the early stage of AI infection could contain the virus on the egg yolk, albumen and the eggshell. The virus can also penetrate cracked or even intact eggshells $(1,2,8)$.

HPAI Virus is present in all secretions and excretions of infected birds. Transmission may be direct inhalation of contaminated aerosol, dust as droplets and droplets nuclei or indirectly via primarily attributed to movement of ingestion of contaminated water, feeds or infected carcasses. Transmission of HPAI between flocks is infected birds and action of humans through movement of feeds/personal equipment and vehicles into or out of contaminated premises. Spread of the disease into counties could be through importation or smuggling of infected poultry and poultry products across the border and through migratory birds (9). The virus is infective for almost all commercial, domestic and wild avian species. Chickens and turkeys are highly susceptible. Ducks and geese though susceptible to infection with all AIV strains but suffer clinical disease from only virulent strain. Other birds are susceptible at varying degree, while Pigs and humans are equally susceptible to infection by the HPAI virus $(1,2,10)$.

\section{Prevalence and distribution of AIV}

AI is an infectious disease of birds caused by type A strains of the influenza virus. The disease, which was first identified in Italy more than 100 years ago, occurs worldwide. In the history of man, it is responsible for the largest pandemics of the last century especially "Spanish flu" pandemic of 1918-1919 which claimed over 40 million lives $(1,10,11)$. From 1998 to 
2002 avian flu pandemic with onset in China has spread round the world resulting in avoidable human death and over 20million chicken culled worldwide $(1,10,11)$. The current outbreak of highly pathogenic AI A(H5N1), which began in Southeast Asia in mid-2003, is the largest and most severe affecting many countries simultaneously. Despite the death and destruction of over 150 million birds, the virus is now considered endemic in many parts of Indonesia and Viet Nam and in some parts of Cambodia, China and Thailand (10-12) . The risk of a pandemic will persist until the disease is controlled in birds, which may take several years.

The first human cases of AI A (H5N1) associated with the current outbreak in birds were confirmed in January 2004 in two children and one adult admitted into hospital in Hanoi with severe respiratory illness who tested positive for this strain. Since then, additional human cases have occurred in several countries, and the clinical spectrum of AI A (H5N1) infection in humans ranges from asymptomatic infection to severe disseminated disease $(2,3,12)$. In the African Region, little is known about the burden of avian influenza because few countries have laboratory capacity for virus isolation. No documented H5N1 infection has been reported in the region. However, an outbreak of H5N2 influenza was reported among ostriches in South
Africa in 2004 and in December 2005 in Zimbabwe (13). In order to address the threat of a pandemic of influenza, in African region, WHO have tried to improve and strengthen laboratory capacities of African counties for influenza and promoting the framework of Integrated Disease Surveillance and Response (IDSR) strategy (13).

There is increasing concern that Africa is at great risk of pandemic influenza. Firstly, as winter sets in Northern hemisphere, migratory birds can bring the deadly virus to Africa. Secondly, with increasing global travel there is a high risk of dissemination of the $\mathrm{H} 5 \mathrm{~N} 1$ virus. Thirdly, the poor health infrastructure in Africa and the nonavailability of vaccines or drugs means that millions of people would be at high risk of pandemic influenza (1-4).

In January 2006 AI infection was reported in Sambawa poultry Farm in Kaduna state of Nigeria and this was confirmed on 7th February 2006 by international office of Epizootic (IOE) reference Lab in Padova, Italy (9). Since then several focal outbreaks in poultry farms have been recorded in 13 Northern states of Nigeria including Abuja, the Federal Capital Territory (FCT). More than 2 million chickens were culled, poultry farms closed down 
and huge some of money paid as compensation to poultry farmers (9). Although, only Four (4) human morbidity cases and one death were recorded in the country (9). This figure probably represents a tip of ice berg because of the prevailing poor reporting and incomplete data that characterized the disease surveillance and notification system in Nigeria and indeed other developing African countries.

\section{Risk factors for AI infection}

Since 1878 when HPAI was first reported in Italy, the infection had spread across all international boundaries due to increase international travels, migratory birds, poultry and products exports, hence assuming a rapid pandemic trend in the last decade with pockets of epidemics world over. AIVs are probably ubiquitous in wild water birds. Pathogenic strains could emerge and cause disease in domestic poultry in any country at any time without warning. The occurrence of human cases creates enormous challenges to the health systems. Laboratory confirmation and management of human $\mathrm{H} 5 \mathrm{~N} 1$ viral infections is technically challenging, expensive, and demanding on human and financial resources. The major risk factors for AI infection and outbreak of HPAI in developing countries can be grouped into 2 :

\section{i. Risk of introduction of AIVs} through migratory birds: Nigeria lies in the East Africa/West Asian and the North Atlantic flyways of the migrating birds, and hence is at risk of the introduction of the virus. Also, increasing trade and human traffic between Nigeria and other countries in South East Africa and South Africa is another major threat to having the infection (9). Expansion of infection zone of Avian influenza due to globalization and relative ease of movement and transportation through porous borders that encourage informal livestock movement, Smuggling / illegal movement of poultry and products from infected countries coupled with inadequate veterinary quarantine facilities and manpower are the major risk factors for introduction of AIV into other countries especially those within the flyways of these migratory birds.

\section{ii. Risk of sustenance and maintenance of HPAI in African} countries: Risk factors under this category are associated with poverty, ignorance and sociocultural believes and practices relating to keeping of pets and domestic animals for economic 
and spiritual purposes. Also, lack or poor implementation of veterinary policies and inefficient public veterinary services are other issues that may encourage sustenance of the virus. Some of the specific factors that arise from above issues are $(1,9)$ :

- Backyard poultry, periurban and urban commercial poultry production with little or no bio-security and constant introduction of new birds from relatively unknown and unverified sources.

- Uncontrolled livestock and poultry movement within and outside the country due to lack of enforcement of animal disease control laws and regulation, including registration and Licensing of poultry farms \& hatcheries

- Increased close contact between poultry and human, and lack of organized poultry marketing which encourage open live poultry markets characterized by interspecies mixing and poor sanitary condition.
- Inadequate experience of most vet and health workers in the recognition \& diagnosing of HPAI.

- Inadequate / lack of funding for veterinary services, compensation of Livestock/ flocks owners in the events of mass culling of birds and poor disposal facilities for culled or dead birds thus encouraging sale and consumption of sick and dead birds

\section{Threats and Consequences of HPAI}

AIVs do not infect humans because of host barriers to infection, such as cell receptor specificities. However, they can occasionally cross the species barrier and directly infect humans, including highly pathogenic strains that have caused fatal disease in humans.(1,2) In 1997, AI A(H5N1) caused an outbreak in domestic poultry in Hong Kong and also infected 18 people and causing 6 deaths $(11,14)$. Since then, other AI outbreaks have resulted in human infections. The outbreaks of highly pathogenic H5N1 avian influenza in poultry which began in Asia in mid-2003 have led to over 120 confirmed human cases of which more than half have died $(1,2)$. The majority 
of cases occurred in previously healthy children and young adults. Historically, the number of deaths during a pandemic has varied greatly. Death rates are largely determined by four factors: the number of people who become infected, the virulence of the virus, the underlying characteristics and vulnerability of affected populations, and the effectiveness of preventive measures.

Presently, avian influenza A (H5N1) is endemic in birds in many parts of the world. The widespread persistence of H5N1 in bird populations poses two main risks to human health. The first is the risk of infection when the virus spreads directly from birds to humans. The second risk, which is of even greater concern, is that there will be increased possibilities for the widely circulating virus to infect humans and possibly re-assort into a strain that is both highly infectious for humans and spreads easily from human-to-human. Such a change could mark the start of a pandemic (15).

One important feature of AIV is the tendency to acquire new properties through adaptation, mutation and recombination that may lead to emergence of a highly virulent virus with many serotypes. The fear is that in nearest future a more highly virulent virus with high possibility of man to man transmission could be produced if the spread of HPAI is not curtailed. The implication of the pandemic nature is such that stringent international and National regulations on Quarantine and veterinary Laws have to be put in place which may affect human movements, poultry and diary products exports. This will invariably affects tourism, international trade and the world economy. For example, over 2million chicken were culled and about 160,000 US dollars paid as compensation to poultry farmers following January / February 2006 outbreaks of AI in Nigeria. These outbreaks also caused loss of jobs due to closure of poultry farms (9). At the global level, it is estimated that 20million chicken were culled causing the world over 2Billion USD loss $(1,2)$. This huge amount of resources could have been used for other health conditions.

Nigeria has an estimated poultry productions of around 140million birds largely concentrated in the Southwestern part of the country and $60 \%$ of these poultry production takes place in small backyard flocks, making families, associates and visitors at risk (9). Large scale commercial farming of poultry occurs mainly in the Northern States where outbreak had been confirmed. Rapid spread of the virus within Nigeria has raised concern over possible spread to neighboring countries in the 
sub-region because of porous borders and restriction of movement of people and poultry are difficult to enforce, thus putting the neighboring countries at risk.

Another implication of HPAI outbreaks and pandemic is that Medical supplies will be inadequate. Vaccines and antiviral drugs (the two most important medical interventions for reducing illness and deaths during a pandemic) will be inadequate in all countries at the start of a pandemic and for many months thereafter. Inadequate supplies of vaccines are of particular concern, as vaccines are considered the first line of defense for protecting populations. On present trends, many developing countries will have no access to vaccines throughout the duration of a pandemic; and because of this, large numbers of deaths will occur $(1,2)$. Economic and social activities will be disrupted due to High rates of illness and worker absenteeism and schools may be shot down. Social and economic disruptions would be worse if workers absenteeism involve people in essential services, such as power, transportation, health and communications.

\section{Prevention and Control}

AIV usually spreads when live birds carrying infection are bought and sold, and by contact of birds with bird droppings on dirty equipment, cages, feed, vehicles or shoes/clothing $(9,16)$. Good hygienic practices (bio-security) is therefore an extremely important safety measure to prevent infection entering domesticated poultry; and all persons keeping or working with poultry should play their role in this. Therefore, promoting and encouraging good health habit would go along way in curtailing the spread of the virus in human population. Health education and awareness campaign to the public, poultry workers, diary products handlers, families with backyard poultry will go a long way to prevent spread of the virus. Specific preventive measures are:

\section{a. Prevention of introduction of HPAI into the country}

This can be achieved by adopting WHO recommended standard measures and strategies which include $(6,16,17)$ :

- Ban on importation of poultry and poultry products from countries where the disease is known to exist especially from the Asian continent

- Adopting effective animal disease surveillance involving 
- high-risk areas such as poultry markets, wet lands and poultry located along known migratory birds routes, poultry abattoirs, borders and targeted farms should be carried out.

\section{- Creation}

or

revitalization of functional National veterinary Quarantine services (NVQS) including immediate rehabilitation and revitalization of existing veterinary quarantine infrastructure, control post and enhancing manpower capabilities.

- Targeted community based training of rural backyard poultry farmers in various aspects of HPAI recognition and control including bio-security procedures applicable to rural small-scale poultry enterprises and the role of animal disease vigilante in the control of HPAI.
- Introduction and enforcement of regulatory policies and requirements for import permits for poultry and poultry products by the Federal authorities or agency concern with live stock services.

- Development of a traceable mechanism for animal and strict monitoring of movement of poultry and poultry products through registration and Licensing of poultry farms, hatcheries and other poultry enterprises.

- Development of a community based, participatory rural livestock and poultry disease surveillance system and integrating it into the existing epidemiological surveillance network.

- Training of veterinarian, auxiliaries, others 
- ategories of poultry

farmers on HPAI

prevention and control

strategies including

aspect of bio-security

and early recognition /

detection of the disease.

- Effective public

enlightenment and

awareness programmes

on HPAI policy and

preventive services

available to the public

\section{b. Specific control measures in Birds} and man

Preventive strategies and activities under this category are $(2,3,9,10,16,17)$ :

- Importation

restriction/ regulation

of birds

- Proper care of poultry farm that include nutrition, vaccination, isolation of infected birds, mass destruction of infected birds and disinfection poultry farms. Control of the disease in birds by early diagnosis \& treatment

- Proper regulation and inspection of poultry

farms and products by

the regulatory

authorities. Food

Hygiene involving

proper meat and

chicken inspection,

farm inspection,

thorough cooking of

meat and chicken.

Avian flu does not

survive high

temperature.

- Prompt response to

disease outbreak in

birds' population e.g.

Proper notification, isolation and veterinary

surveillance.

- Observing universal

precautions and

passionate personal

hygiene among poultry workers before and after handling poultry birds and poultry products. Provision and use of personal protective measures (like apron, coats, gloves, boots, goggles and masks etc) for high risk groups like poultry workers, vet officers. 
- Immunization against HPAI among people at high risk, e.g. veterinary officers, poultry farmers \& workers, Diary handlers etc.

- Avoidance of unnecessary contact and proximity to poultry area especially among backyard poultry, free ranging flock and birds aspects.

\section{c. Early Diagnosis \& Screening}

Diagnosis involves clinical signs and symptoms with confirmatory Laboratory tests which include: virus isolation and gene sequence detection at the National veterinary laboratory (where available) and further characterization of isolates like heamoglutinin and neuraminidase typing could be done at national Lab and other regional and world reference laboratories. The WHO recommended standard procedures for the screening and diagnosis of suspected human influenza A H5NI cases are: $(9,16,18-$ 20)

\section{Surveillance case definition for community and Health workers}

definition consists of fever, cough and history of contact with sick / dead birds or history of contact with another person with similar complaints. Members of the community are expected to report and/ or send a. Rapid antigen detection using any of the following tests: Immuno-fluorescence assay which is a widely used, sensitive method for diagnosis of influenza A and B virus infections and five other clinically important respiratory viruses; and Enzyme immunoassay for influenza A nucleoprotein (NP). The results of these tests can be obtained within 30 minutes.

b. Virus culture provides results in 2-10 days. Both shell-vial and standard cell-culture methods may be used to detect clinically important respiratory viruses. Positive influenza cultures may or may not exhibit cytopathic effects but virus identification by immuno-fluorescence of cell cultures or haemaglutinationinhibition (HI) assay of cell culture medium (supernatant) is required.

c. Polymerase chain reaction 
(PCR) and Real-time assays. Primer sets specific for the haemaglutinin (HA) gene of currently circulating influenza $\mathrm{A} / \mathrm{H} 1, \mathrm{~A} / \mathrm{H} 3$ and $\mathrm{B}$ viruses are becoming more widely used. Results can be available within a few hours from either clinical swabs or infected cell cultures.

Samples required for birds are tracheal ad cloacae swabs, faecal samples, and paired sera from live birds. Intestinal contents, samples of trachea, lungs, air sacs, spleen, kidneys, brain, liver, pancreas and heart can also be used. Laboratory diagnosis for birds includes antigen detection through Immunoflourescence and Direct antigen Flu A tests (Directigen TM fluA test). This is followed by Virus Isolation and identification using Reverse transcriptase polymerase chain reaction (PT - PCR), Virus characterization through Haemaglutinin typing, neuraminidase typing, gene sequence detection and Serological diagnosis using Haemaglutination Inhibition test (HIT), Agar Gel Immuno-diffusion test (AGID), Competitive Enzyme linked Immunosorbent Assay (C-ELISA). Infected animals could be differentiated from vaccinated by serological test called Differentiating Infected from Vaccinated Animal System (DIVA).cases meeting this definition to nearest health facility. Health care workers must be at alert to suspect cases of avian flu by using health facility based case definition which is (9):

- Fever $>38^{\circ} \mathrm{C}$ with History of exposure in last 7 days before onset of illness and one or more of the following symptoms / signs: Cough, sore throat, Breathing difficulties.

- Any person requiring hospitalization for an influenzalike illness and living in, or coming from an area with avian influenza outbreak..

- Any person having died of an unexplained respiratory illness.

\section{Notification of suspected cases}

Any case that meets the suspected case definition should be immediately notified to the Disease Surveillance and Notification Officer (DSNO) at the district, regional or national health office using duly completed Immediate case based reporting form (IDSR 001) and must be sent by the fastest means (e.g. fax, email, radio, telephone etc). Following this reporting, a Rapid Response team would be dispatched to conduct detailed investigation and reassessment of the suspected cases and fresh specimen collected. The team also arrange for the transfer of patient to 
avian influenza treatment centre (if

vailable) using dedicated vehicle; and help the local / district health unit / facility or department in producing plan to identify and follow up close contacts.

\section{Laboratory diagnosis of AI and} HPAI infection in birds and man

Laboratory identification of human influenza A virus infections is commonly carried out by direct antigen detection, isolation in cell culture, or detection of influenza-specific RNA by reverse transcriptase-polymerase chain reaction. The optimal specimen for influenza A virus detection is a nasopharyngeal aspirate obtained within 3 days of the onset of symptoms. Nasopharyngeal swabs and other specimens including serum can also be used. Assays available for the diagnosis of influenza A virus infections include (18-22):

\section{d. Case management}

Case management at the hospitals (or avian influenza treatment centre) includes patient isolation, barrier nursing and maintenance of precautionary measures. The patients and family must be educated on the risk of transmission and the need to notify health Authorities of other suspected cases.
Standard precautions should be used for all patients receiving care in hospitals, regardless of their diagnosis or presumed infection status. Some of the precautionary measures include $(19,23-25)$ :

i) Hand washing with either a plain or antimicrobial soap and water to prevent possible selfinoculation of the nose, mouth, and conjunctivae and the transfer of micro-organisms to the environment or other patients by contaminated hands. The use of an alcoholbased hand rub for routine hand antisepsis is recommended. Many studies have demonstrated that influenza, an enveloped virus, is susceptible to alcohols when tested in vitro and in vivo testing with a 95\% ethyl alcohol.

ii) Use of gloves when touching mucous membranes and nonintact skin, blood, body fluids, secretions, excretions, and contaminated items. Change gloves between tasks and procedures on the same patient after contact with material that may contain a high 
concentration of micro-

organisms. Remove gloves

promptly after use and wash

hands immediately to avoid

transfer of micro-organisms to

other patients or environments.

iii) Wear mask and eye protection or a face shield to protect mucous membranes of the eyes, nose, and mouth during procedures and patientcare activities that are likely to generate splashes or sprays of blood, body fluids, secretions, and excretions.

iv) Wearing of gown: a clean, non-sterile gown is required to protect skin and prevent soiling of clothing during procedures, patients' care and other activities that are likely to generate splashes or sprays of blood, body fluids, secretions, or excretions. Soiled gown should be removed as promptly as possible and wash hands to avoid transfer of microorganisms to other patients or environments.

e. Drug treatment and use of vaccine

Two drugs (in the neuraminidase inhibitors class), oseltamivir (commercially known as Tamiflu) and zanamivir (commercially known as Relenza) are available; and both can reduce the severity and duration of illness caused by seasonal influenza. The choice of drug may depend on its availability and cost. The efficacy of the neuraminidase inhibitors depends on their administration within 48 hours of onset of symptom (26). For cases of human infection with H5N1, the drugs may improve prospects of survival, if administered early, but clinical data are limited. The dosage treatment with a neuraminidase inhibitor such as oseltamivir is $75 \mathrm{mg}$ orally, twice daily for 5 days. Reference must be made to manufacturer's instruction for children dosage $(9,16)$. Supportive cares with oygen, antibiotics and antipyretics may be required. Administration of salicylates (such as aspirin) in children less than 18 years of age must be avoided because of the risk of Reye syndrome, rather paracetamol or ibuprofen can be used $(9,16,26)$.

Vaccines effective against a pandemic virus are not yet 
available. Vaccines are produced each year for seasonal influenza but will not protect against pandemic influenza. Although, vaccine against the $\mathrm{H} 5 \mathrm{~N} 1$ virus is under development in several developed countries, none is ready for commercial production. Some clinical trials are now under way to test whether experimental vaccines will be fully protective and to determine whether different formulations can economize on the amount of antigen required, thus boosting production capacity $(16,27)$.

\section{f. Discharge policy}

Studies are ongoing for better understanding of viral excretion patterns in humans infected with the influenza $\mathrm{A}(\mathrm{H} 5 \mathrm{~N} 1)$ viruses. However, WHO recommends that adult patients remain in hospital (if admitted) or confined to a location ( unhospitalised) for 7 days after resolution of fever. Previous human influenza studies have indicated that children younger than 12 years can shed virus for 21 days after onset of illness $(5,27)$. Therefore, infection control measures for children should ideally remain in place for this period. Where this is not feasible the family should be educated on personal hygiene and infection control measures; and the affected child should not attend school during this period.

\section{ACKNOWLEDGEMENTS}

We thank, Prof. D. B. Parakoyi, of the Dept of Epid \& Community Health, University of Ilorin Teaching Hospital, Nigeria for going through the manuscript and providing useful comments. We also acknowledge the support of the World Health Organization, Kwara State office in Nigeria for providing relevant literatures / documents.

\section{Reference:}

1. Zeitlin G, Maslow M. Avian Influenza. Curr Infect Dis Rep 2005; 7:193-199

2. WHO; Avian influenza A (H5N1) infection in humans. $\mathrm{N}$ Engl J Med 2005; 353: 1374-85

3. Hayden F, Croisier A. Transmission of Avian Influenza Viruses to and between Humans. J Infect Dis 2005; 192: 1311-4

4. Nicholson KG, Wood JM, Zambon M (2003). Influenza. Lancet, 362:1733-1745 
5. World Health Organization

(WHO); Selected guidelines on influenza

H5N1.

http://www.who.int/csr/disea se/avian_influenza/guidelines

/en/

6. International Food Safety Authorities

Network

(INFOSAN); Highly pathogenic

H5N1 avian influenza outbreaks in poultry and in humans: Food safety implications; INFOSAN Information Note No. 7/2005 (Rev 1. 5 Dec) Decemmber 2005; pg $1-4$

7. Lu H, Castro AE, Pennick K, et al. Survival of avian influenza virus H7N2 in SPF chickens and their environments. Avian Dis 2003; 47: 1015-21

8. Swayne D., Beck J. (2004); Heat inactivation of avian influenza and Newcastle disease viruses in egg products. Avian Pathology 33(5), 512-518

9. Federal Ministry of Agric (Dept of Live stocks \& pest control services); Report of technical committee on the prevention \& control of HPAI in Nigeria; December 2005, p 5-18.

10. Horimoto T, Kawaoka Y
Influenza: lessons from past pandemics, warnings from current incidents. Nat Rev Microbiol 2005; 3:591-600

11. Chan PK. Outbreak of avian influenza A (H5N1) virus infection in Hong Kong in 1997. Clinical Infectious Diseases, 2002, 34:S58-S64

12. WHO. Inter-countryconsultation: influenza A/H5N1 in humans in Asia: Manila, Philippines, 6-7 May. 2005

13. WHO Regional Director for Africa, Opening remarks at the regional meeting of experts on pandemic of avian influenza. January 2006; Brazzaville DRC

14. Olsen S.J., et al. (2005) Poultryhandling practices during avian influenza outbreak, Thailand. Emerging Infectious Diseases, Vol. 11, No. 10

15. Ungchusak K, Auewarkul P, Dowell SF et al; Probable person to person transmission of avian influenza A (H5N1). N. Engl J. Med 2005; 352: 33 - 40.

16. WHO (Western Pacific regional office): Avian Influenza, 
17. including Influenza A (H5N1), in Humans: WHO Interim Infection Control Guidelines for Health Care Facilities. Published on 9 $9^{\text {th }}$ February, 2006; by WHO WPRO, Manila Philippine. Pg 6-43

18. European Commission; Introducing Community measures for the control of avian influenza. Council Directive 92/40/EEC of 19 May 1992 Official Journal of the European Union, L 167, 22.6.1992, p.1

19. WHO, Recommended laboratory tests to identify avian influenza A virus in specimens from humans $\mathrm{WHO}$ Geneva, June 2005. http://www.who.int/csr/disea se/avian_influenza/guidelines /referencelabs/en/index.html

20. Yuen KY, Chan PK, Peiris M, et al. Clinical features and rapid viral diagnosis of human disease associated with avian influenza A H5N1 virus. Lancet 1998; 351:467-71

21. Fouchier RA et al. (2000); Detection of influenza A viruses from different species by PCR amplification of conserved sequences in the matrix gene.
Journal of Clinical Microbiology, 38:4096-4101.

22. Lee CW, Suarez DL (2004). Application of real-time RTPCR for the quantitation and competitive replication study of H5 and H7 subtype avian influenza virus. Journal of Virological Methods, 119: 151158.

23. Wright KE et al. (1995). Typing and sub-typing of influenza viruses in clinical samples by PCR. Journal of Clinical Microbiology, 33:1180-1184.

24. Garner J. Guideline for Isolation Precautions in Hospitals. Infect Control Hosp Epidemiol; 1996; 17: $53-80$

25. Suarez D, Spackman E, Senne D, Bulaga L, Welsch A and Froberg K. The effect of various disinfectants on detection of avian influenza virus by real time RT-PCR. Avian Dis 2003;47:1091-1095

26. Swayne D., Beck J. (2005) Experimental study to determine if low-pathogenicity and high-pathogenicity avian 
27. influenza viruses can be present in chicken breast and thigh meat following intranasal virus inoculation. Avian Diseases 49:81-85

28. Hayden FG, Atmar RL, Schilling $\mathrm{M}$, et al. Use of the selective oral neuraminidase inhibitor oseltamivir to prevent influenza. N Engl J Med 1999; 341:1336-43.
29. World Health Organization (WHO); Guidelines on the use of vaccines and antivirals during influenza pandemics. Geneva, 2004. (Document WHO/CDS/CSR/RMD/2004.8

.)
Visit our website: http//www.ajol.info/journals/ajcem 